\title{
GPS WIRELESS SENSOR NETWORK FOR MONITORING QUASI-STATIC DISPLACEMENT
}

\author{
Masayuki SAEKI ${ }^{1}$, Mai SAWADA 2 , Yukio SHIBA ${ }^{3}$ and Kenji OGUNI ${ }^{4}$ \\ ${ }^{1}$ Member of JSCE, Associate Professor, Dept. of Civil Eng., Tokyo University of Science \\ (Yamazaki 2641, Noda-shi, Chiba 278-8510, Japan) \\ E-mail: saeki@rs.noda.tus.ac.jp \\ ${ }^{2}$ Member of JSCE, Civil Eng. Res. Inst., Technology Center, Taisei Corp. \\ (Nase-cho 344-1, Totsuka-ku, Yokohama-shi 245-0051, Japan) \\ E-mail: swdmi-00@pub.taisei.co.jp \\ ${ }^{3}$ Fellow Member of JSCE, Civil Eng. Res. Inst., Technology Center, Taisei Corp. \\ (Nase-cho 344-1, Totsuka-ku, Yokohama-shi 245-0051, Japan) \\ E-mail: shiba@ce.taisei.co.jp \\ ${ }^{4}$ Member of JSCE, Associate Professor, Dept. of System Design Eng., Keio University \\ (3-14-1 Hiyoshi, Minato-ku, Yokohama-shi 223-8522, Japan) \\ E-mail: oguni@sd.keio.ac.jp
}

\begin{abstract}
This paper presents a system for monitoring quasi-static displacements, named GWSN for "GPS wireless sensor network". This system consists of the technologies of a low-cost L1 GPS (Global Positioning System) and wireless sensor network. The low-cost sensor node and cable-less system enable us to deploy sensors in dense numbers over a vast area. In general, displacement monitoring using GPS has a trade-off relationship between energy consumption and accuracy of estimation. Measuring continuous data and applying a trend analysis or Kalman filter outputs a highly accurate displacement estimation but also needs much energy dissipation. To solve the trade-off relationship, a data compression method and a static GPS positioning analysis method were developed and implemented in the system. In these methods, the assumption of quasi-static displacement was taken into account. A prototype system was developed and experiments were conducted in a quarry site and in an ideal condition using the developed prototype. The experimental results show that the prototype system works without serious system trouble in outdoor environments and has a great potential to measure displacements with sub-centimeter accuracy.
\end{abstract}

Key Words : GPS, wireless sensor network, quasi-static displacement, monitoring

\section{INTRODUCTION}

\section{(1) Background and objectives}

Monitoring displacements of embankments, slopes or reclamation land is important in construction sites for controlling quality and ensuring safety. In recent years, displacement monitoring has been automated rather than done through traditional survey work. In the existing system, a high-performance GPS (Global Positioning System), a total station and/or a laser displacement meter are used as a displacement sensor. The sensors are generally connected to cables and remotely controlled.

The existing system is capable of measuring displacements very accurately. However, it is unrealistic to deploy the sensors in dense numbers over a vast area because the cost of installation and maintenance is quite expensive. Therefore, we have been developing a displacement monitoring system which uses a wireless sensor network and an affordable L1 GPS receiver that is connected to a small patch antenna generally used for mobile navigation systems. We call this system GWSN (GPS Wireless Sensor Network). This inexpensive wireless sensor system allows us to monitor displacements with high spatial resolution.

Application of wireless sensor networks is one of the hot topics in the research field of civil engineering. For example, research on an application for measuring vibration of civil infrastructures and on an effective data collection method in maintenance

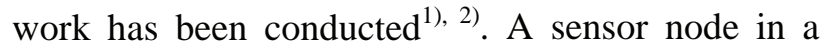


wireless sensor network consists of physical sensors, a small wireless communication device, a micro-controller and a battery. Sensor nodes organize a local network autonomously, collect measurements and analyze them ${ }^{3}$. So the GWSN might be a typical application of a wireless sensor network. The general merits of wireless sensor networks are summarized as follows:

1) Because it is cable-less, the installation cost can be minimized and there is no possibility of data loss due to cable cut.

2) Since sensor nodes are separately controlled, malfunction of a sensor node does not influence the whole system.

3) When a sensor node breaks down, the system is easily recovered by replacing the broken node with another one.

In order to develop a cable-less system, a decrease in energy consumption is required, because high energy consumption requires usage of power supply cables or a large solar battery. Intermittent action is the best solution for minimizing energy consumption. But it leads to shortage of data and diminishes the accuracy of displacement monitoring. Therefore, the system has a trade-off relationship between accuracy and energy consumption.

To solve the trade-off relationship, we have developed a data compression method and a static GPS positioning analysis method and implemented them in the prototype system. We also conducted experiments in an open quarry site and in an ideal condition to investigate the performance of the prototype system.

\section{(2) Related research}

Displacement monitoring systems using a lowcost L1 GPS receiver have been developed by many researchers for monitoring landslides or volcanoes. For example, Shimizu et al. developed a displacement monitoring system in which L1 GPS receivers were networked with cables, and applied their system to a landslide site. In their system, the trend model was adopted to improve the accuracy of the measurement results and achieve sub-centimeter accura$\mathrm{cy}^{4), 5), 6)}$. Seynat et al. developed a system consisting of a central server and sensors equipped with a radio -link, data storage and L1 GPS receiver ${ }^{7}$. In those researches, demonstrations were conducted by distributing sensors several kilometers away from a base station.

The system presented in this paper differs from those researches in our use of the wireless sensor network, which enables us to develop a cable-less, low energy consumption, small in size and affordable system.

Data compression is one of the important tasks in a wireless sensor network, because a low-power and narrow-bandwidth wireless communication device is employed $^{3)}$. Data compression has many merits, such as short communication time, low packet-loss rate and low energy consumption. Therefore, there are many researches on data compression ${ }^{8}$. For example, a structural sensing system has been studied in which distributed sensor nodes locally communicate to each other and share their collected data. After analyzing the collected data using the processor mounted on the sensor nodes, the sensor nodes send only the analyzed results to the central server ${ }^{2}$.

In GWSN, the data cannot be analyzed on the sensor node because the estimation of displacement needs both data of sensor nodes located at a measuring point and data located at a reference point. So all the data received by the sensor nodes should be transmitted to the central server. In the proposed method, the data compression is achieved by extracting the essential data from raw GPS data. Required data for estimating the relative position of sensors with accuracy of a few centimeters is described in the compact data transmission standard ${ }^{99}$. The CMR (Compact Measurement Record) format is commonly used ${ }^{10)}$. In the newly developed data compression method implemented in GWSN, most of the essential data which is truly needed for estimating quasi-static displacement is still extracted from the CMR format.

\section{(3) Contents}

The authors developed the required elemental technologies, demonstrated the performance in cold snowy conditions ${ }^{12)}$, implemented the prototype system and conducted an experiment on displacement monitoring in an open quarry site. Currently, we are improving the system for practical applications.

This paper describes the developed elemental technologies and shows the results obtained from the experiments conducted in an open quarry site and in an ideal condition. In the second section, an overview of the GWSN and the system requirements are explained. The elemental technologies, i.e., displacement estimation analysis method and data compression technique, are presented in the third and fourth sections, respectively. In the fifth section, the results of the experiments conducted using the prototype system are described. Finally, we summarize the outcomes obtained so far and point out the remaining problems to be solved. 


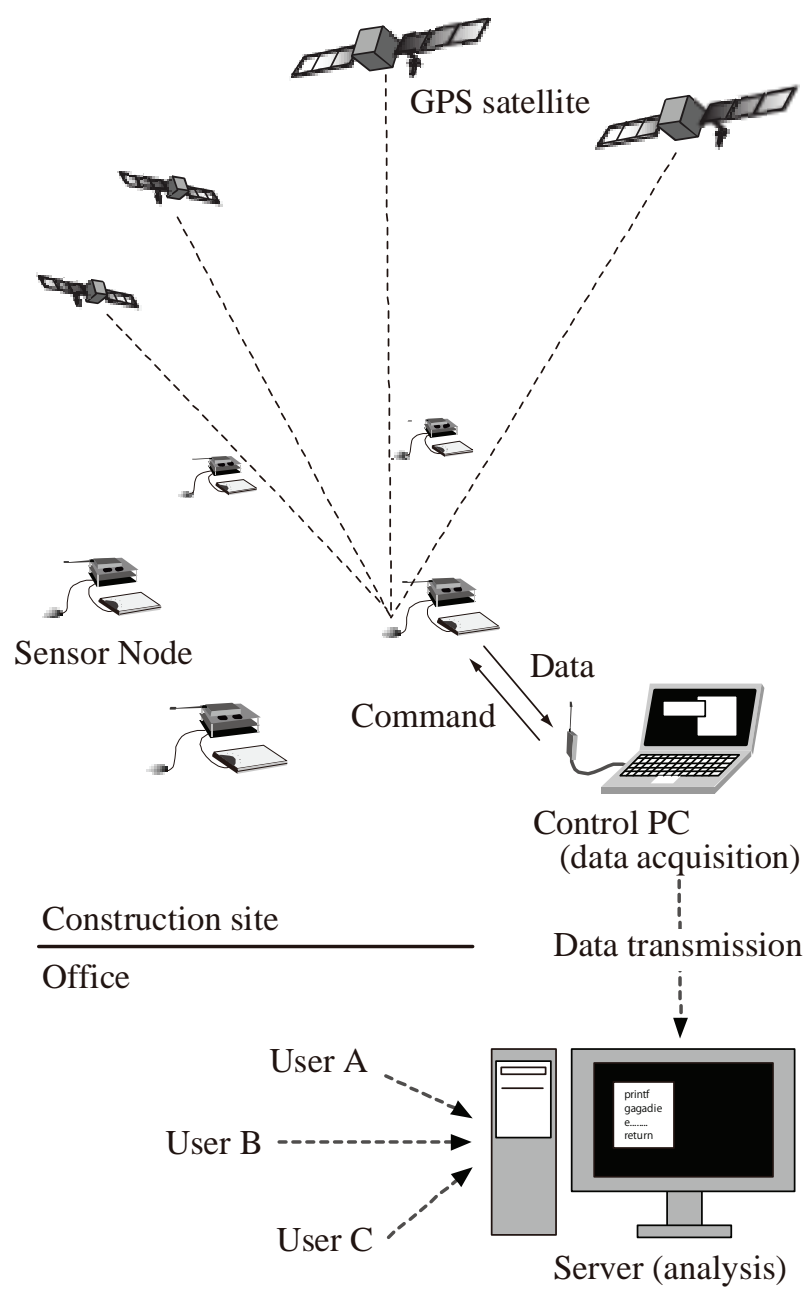

Fig.1 Schematic view of GWSN.

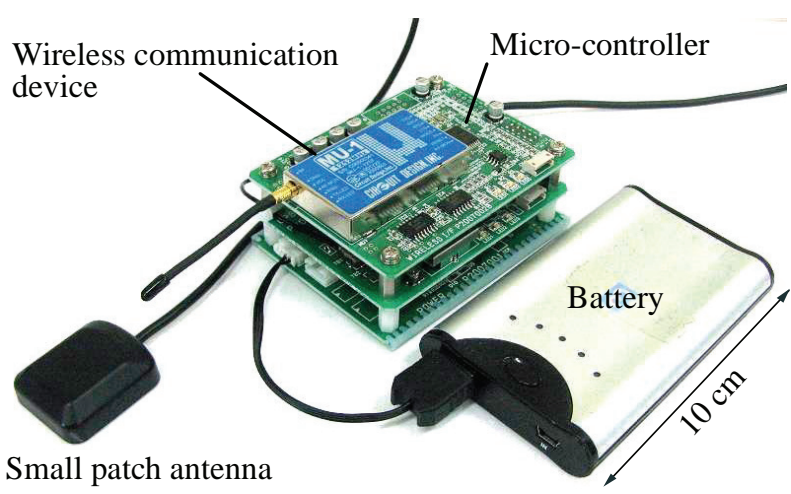

Photo 1 Prototype of a sensor node.

\section{GPS WIRELESS SENSOR NETWORK}

\section{(1) Configuration of the system}

Fig.1 illustrates a schematic view of the GWSN. At a target field, the control PC and many sensor nodes are deployed. The prototype of the sensor node is shown in Photo 1. The sensor node receives raw GPS data, makes data packets and sends them to the control PC according to the command from the control PC. The control PC transmits the data sets to the central server through internet or a network having strong communication. Upon receiving the data sets, the central server analyzes them and creates a database of estimated displacement. Users are allowed to access the monitoring results via internet. At present, the prototype system shown in Fig.1 has been developed and demonstrated to work with no serious problem. In this prototype, the star topology is applied in the wireless communication between the control PC and sensor nodes to make it easy to develop a simple yet robust system. Recently, we have been trying to implement the tree topology for expanding the area of displacement monitoring.

The sensor node, shown in Photo 1, has a specified low-power radio module, a micro-controller, a low-cost L1 GPS receiver connected to a small patch antenna and a small battery. MU1 (Circuit Design Co. Ltd.) is used in the prototype as a wireless communication module. The bandwidth is only 6800bps and is much slower than that of IEEE802.15.4 (Zigbee). However, it has a relatively long communication range, over 600 meters. (Because of the recent growing technology, Zigbee has also achieved a long communication range. So we are planning to apply Zigbee to be compatible with our system.) As a low-cost L1 GPS receiver, Furuno GT8032 is used on the prototype. A small patch antenna which is generally used for car navigation and never for accurate positioning is connected to the receiver. The wireless communication module and an L1 GPS receiver are controlled by a micro-controller. In the prototype, PIC16F877A (a middle-range series of Microchip Technology, Inc.) is used as the controller. The micro-controller also controls the power supply to the L1 GPS receiver and wireless communication module through the level converters. The level converters output both $3.3 \mathrm{~V}$ and $5.0 \mathrm{~V}$ electric power as necessary. For the small battery, a lithium-ion battery pack or a rechargeable nickel-metal hybrid battery is employed. This is commonly used for a portable game player or a music playback device.

\section{(2) Sequence of operations}

The operation flow of the sensor node is described in Fig.2. The control PC broadcasts a command every 100 milliseconds. Most of the time the control PC keeps broadcasting the sleep command. When the sensor node receives the sleep command, it turns all devices mounted on the circuit board off and goes into sleep mode to minimize energy consumption. The sleep mode continues for $T_{s}(=180)$ seconds. In the sleep mode, the sensor node runs with the internal clock, which depends on the temperature, and the 


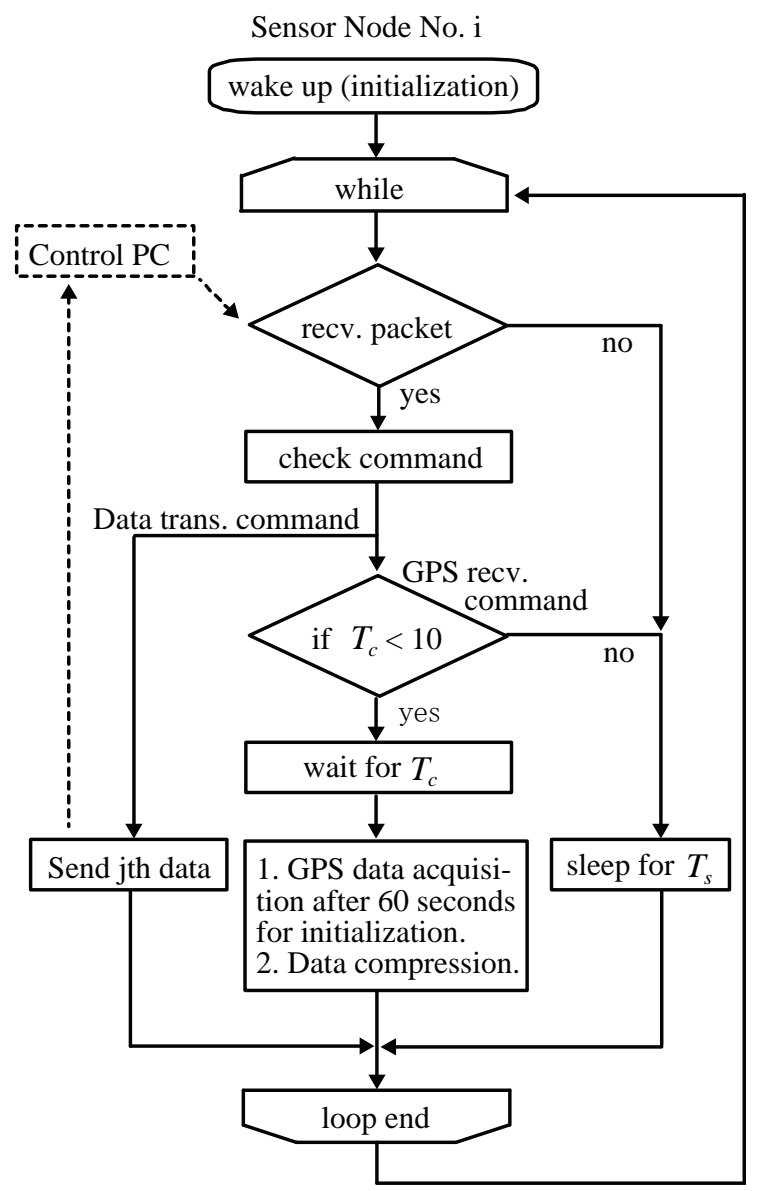

Fig.2 Operation flow of sensor node.

accuracy is very low.

The control PC starts to broadcast the countdown command 10 minutes before the observation time. When the sensor node receives the command, it checks the value of countdown $T_{c}$. If the value is greater than 10 seconds, it goes into sleep mode again. In this case, the sleep time is determined by the following equation.

$$
T_{s}=\max \left(T_{c} / 2,10\right)
$$

Since the internal clock of the micro-controller is inaccurate, it is not able to count time correctly. So in this system, the sensor node sleeps only for half of $T_{c}$ to start data collection at the expected time.

At the start of the observation time, the sensor node turns the L1 GPS receiver on and initializes it. Since the GPS receiver is off most of the time in this system, it starts in the cold start mode. In this case, the receiver first has to search for the satellite's signal and analyze the accurate GPS time and the approximate position of the receiver. It takes at least 30 seconds and generally needs much more time to find out the carrier waves of all available satellite vehicles. In the current system, the initialization time is

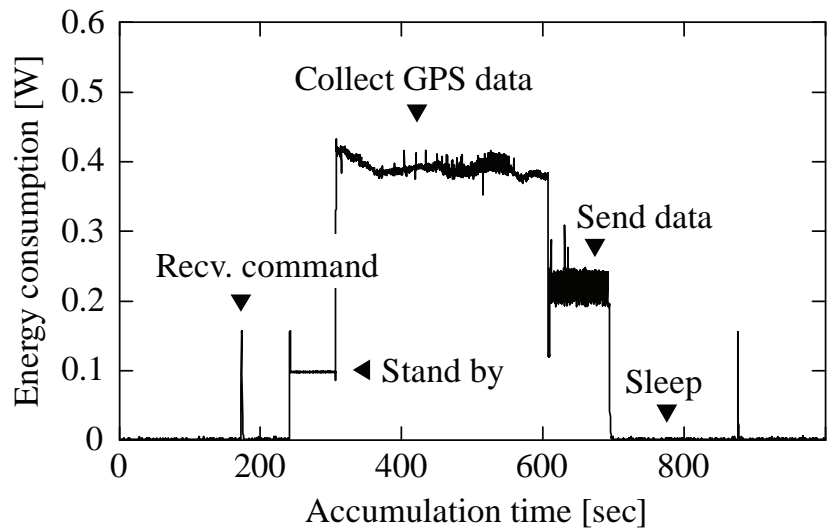

Fig.3 Temporal variation in energy consumption ${ }^{16)}$.

set to be 60 seconds and the GPS data received during the initialization is thrown away. After the initialization, the micro-controller receives data sets from the GPS receiver and saves them in its memory in compressed data format. After the scheduled observation period, the sensor node goes into sleep mode again.

After observation, the control PC broadcasts the data transmission command, which requests the $i$-th sensor node to send the $j$-th epoch data back to the control PC. If the control PC receives the $j$-th epoch data, it adds one to the epoch number $j+1$ and broadcasts the same command again. After getting the total data from a sensor node, the control PC adds one to the ID number of the sensor node. If the control PC cannot get the total data from the sensor node in a certain period of time, it quits the sensor node and tries to the next one. This communication protocol is able to gather all data sets if no serious communication problem occurs.

The control PC transmits the data sets to the central server through the internet after gathering all data from sensor nodes. The central server estimates displacements of sensor nodes and creates the database.

\section{(3) Energy consumption of the sensor node}

Fig.3 illustrates the temporal variation in energy consumption measured using the prototype of a sensor node. As shown in the figure, the sensor node needs the largest electric current when it receives GPS data, which current is about $400 \mathrm{~mW}$. The next largest value is about $220 \mathrm{~mW}$, which happens when the wireless communication module sends data to the control PC. These facts indicate that it is very important to decrease the work duration of the GPS and wireless module. If the GPS receiver is kept on, the lithium-ion battery shown in Photo 1 (12.48Wh) lasts about 30 hours. 


\section{(4) Requirements of the system}

The GWSN is designed to monitor quasi-static displacements with high spatial resolution over an area of one kilometer squared. This restriction gives some important constraints to the development of this system. The following constraints are taken into account:

1) The baseline between measuring and reference points is shorter than that of the usual GPS relative positioning. In this case, the effects of atmospheric delay and ionospheric delay are considered to be negligible in the analysis of static GPS positioning.

2) The GPS observation period should be restricted to a short one to save battery energy for long-term operation.

3) Because of the monitoring of quasi-static displacement, the daily changes are considered to be very small, for example a few millimeters a day.

\section{(5) Component technologies to be developed}

The above condition 2) requires a component technology which enables us to estimate the displacement accurately using data of short length. In general, it seems effective for improving accuracy to analyze continuous data with Kalman filter or trend analysis to suppress noise influence. However, a longer period of data acquisition needs much more energy to run the GPS receiver. To limit energy consumption, a short observation period is a necessary constraint. Therefore, an analysis method which is able to estimate the displacement accurately with a short data length is required.

To minimize energy consumption, it is also important to compress the data size of wireless communication. Thus a data compression method is also required.

From the above discussion, we need to develop the following component technologies:

1) A displacement analysis method which estimates the relative position of sensors accurately with short data length.

2) A data compression method for raw GPS data.

\section{DISPLACEMENT ANALYSIS METHOD}

\section{(1) Observation equation and noise behavior}

The phase of the carrier wave omitted from a satellite vehicle $k$ and received at GPS receiver $i$ at time $t$ is described as $\phi_{i}^{k}(t)$ in this paper. Since the noises due to the troposphere and ionosphere are negligible in the relative positioning analysis, the Double-Difference (DD) observation equation can be modeled like the following equation ${ }^{13)}$ :

$$
\phi_{i j}^{k l}(t)=\rho_{i j}^{k l}(\mathbf{x}, t)+\lambda N_{i j}^{k l}+\Delta_{i j}^{k l}+\varepsilon_{i j}^{k l}(t)
$$

where $*_{i j}^{k l}$ represents the DD values of the GPS satellites $k, l$ and the sensor nodes $i, j$ :

$$
*_{i j}^{k l}=*_{i}^{k}-*_{j}^{k}-\left(*_{i}^{l}-*_{j}^{l}\right)
$$

The term $\rho_{i j}^{k l}(\mathbf{x}, t)$ is the DD range between satellites and the nodes, $\mathbf{x}$ is the relative position vector of a sensor node, $\lambda$ is the wavelength of the $\mathrm{L} 1$ carrier wave, $N_{i j}^{k l}$ is the DD integer ambiguity, $\Delta_{i j}^{k l}$ is the DD antenna noise and $\varepsilon_{i j}^{k l}(t)$ is the random noise.

Because of the quasi-static displacement condition and the 20-centimeter wave-length of L1 carrier waves, once the initial relative position is successfully determined, the DD integer ambiguity on the right-hand side of equation (2) can be easily resolved. Therefore, in the following discussion, we consider the DD integer ambiguity to be involved in the DD carrier phase $\phi_{i j}^{k l}(t)$.

The antenna noise $\Delta_{i j}^{k l}$, which is caused by the multi-path, behaves almost in the same manner as when the surrounding conditions and the geometry of satellites and a sensor are the same. In addition, the GPS satellites go around almost the same orbit every 23h56m. So if we observe carrier phases every 23h56m, the DD carrier phases include almost the same antenna noises. This fact indicates that the antenna noise can be canceled. Therefore, in the following discussion, we consider that the antenna noises $\Delta_{i j}^{k l}$ are included in the random noise $\varepsilon_{i j}^{k l}(t)$.

Here, we express the initial position of sensor node $\mathbf{x}_{0}$ and displacement $\Delta \mathbf{x}$. The position of the sensor node can be expressed as

$$
\mathbf{x}=\mathbf{x}_{0}+\Delta \mathbf{x}
$$

Substituting equation (4) into equation (2) and applying Taylor expansion with respect to $\mathbf{x}_{0}$ yields the following linearized equation.

$$
\phi_{i j}^{k l}(t)-\rho_{i j}^{k l}\left(\mathbf{x}_{0}, t\right)=\nabla \rho_{i j}^{k l}\left(\mathbf{x}_{0}, t\right) \cdot \Delta \mathbf{x}+\varepsilon_{i j}^{k l}(t)
$$

Gathering equation (5) for available satellites yields simultaneous equations. The displacement vector $\Delta \mathbf{x}$ can be estimated by applying the least-squares method to the simultaneous equations.

\section{(2) Linear approximation of the corrected DD carrier phase}

It is known that the term $\nabla \rho_{i j}^{k l}\left(\mathbf{x}_{0}, t\right)$ on the right- 
hand side of equation (5) can be approximated as a linear function of time. In addition, in an actual situation, the amount of displacement $\Delta \mathbf{x}$ is at most one meter and the displacements are constants in a short period of time. Therefore, the left-hand side of equation (5) can be expressed as a linear function of time with a very small tangent ${ }^{14)}$.

$$
u_{i j}^{k l}(t)=\phi_{i j}^{k l}(t)-\rho_{i j}^{k l}\left(\mathbf{x}_{0}, t\right)
$$

In the proposed method, the displacement $\Delta \mathbf{x}$ is not estimated by solving equation (5) directly. First, the approximate linear function, $u_{i j}^{k l}(t)=a t+b$, is estimated for all DD observation equations, and the values of $u_{i j}^{k l}(t)$ corresponding to the first and last epoch are calculated ${ }^{15)}$. Gathering the equations calculated for available satellites, we obtain the following simultaneous equation:

$$
\mathbf{U}(t)=\mathbf{A}(t) \Delta \mathbf{x}+\mathbf{e}(t)
$$

where $\mathbf{U}(t)$ is the observation vector whose components are $u_{i j}^{k l}(t) ; \mathbf{A}(t)$ is the design matrix which depends only on the satellite constellation and sensor position; and $\mathbf{e}(t)$ is the noise vector. Solving equation (7) corresponding to the first and last epoch through the least-squares method yields the following solution:

$$
\begin{aligned}
& \Delta \mathbf{x}=\mathbf{G}^{-1}\left(\mathbf{A}^{T}\left(t_{s}\right)\right. \mathbf{R}^{-1}\left(t_{s}\right) \mathbf{U}\left(t_{s}\right) \\
&\left.+\mathbf{A}^{T}\left(t_{e}\right) \mathbf{R}^{-1}\left(t_{e}\right) \mathbf{U}\left(t_{e}\right)\right) \\
& \mathbf{G}=\mathbf{A}^{T}\left(t_{s}\right) \mathbf{R}^{-1}\left(t_{s}\right) \mathbf{A}\left(t_{s}\right) \\
&+
\end{aligned}
$$

where $t_{s}$ and $t_{e}$ represent the time of the first and last epoch, respectively. $\mathbf{R}(t)$ is the variance-covariance matrix of the observation vector.

The accuracy of displacement estimated by the above method is relatively improved from the conventional method, especially in cases of short data length $^{15)}$.

\section{(3) Segmentation of observation period}

The accuracy of displacement estimated by solving equation (8) depends on the regularity of coefficient matrix $\mathbf{G}$ and the noises included in the observation vector $\mathbf{U}(t)$. Therefore, in order to improve the accuracy, there is no choice except to reduce the noise from $\mathbf{U}(t)$ or improve the regularity of coefficient matrix G. In order to reduce the amount of noise, a high-performance antenna and receiver are needed, which are also expensive. On the other hand, the improvement of regularity of coefficient matrix $\mathbf{G}$ can be achieved by selecting a favorable observation schedule.

The coefficient matrix $\mathbf{G}$ is determined by the geometry of the sensor node and satellite constellation. If the condition number of $\mathbf{G}$ is small enough, the accuracy deterioration due to noise can be minimized. So, in GWSN, we divide the observation time into segments so that the satellite constellation corresponding to each segment becomes as different as possible ${ }^{16)}$.

\section{DATA COMPRESSION}

In the prototype system, we use the wireless communication device with very narrow bandwidth. The data transmission time $T$ [ms] of $n$ bytes data is estimated by the following equation ${ }^{17)}$ :

$$
T=(n+15) \frac{10000}{b r}+(n \times 1.04)+34
$$

where $b r$ is the baud rate of the serial communication between the micro-controller and wireless communication device and is set at 9800 bps in the prototype.

The GPS receiver mounted on the prototype of the sensor node outputs raw GPS data at 266 bytes per second in Furuno Binary Format. If the data is logged for 4 minutes, the total amount of data is about 63.8 kbyte. To send the whole data to the control PC, it might need 98.7 seconds, which can be estimated using equation (10). In the actual communication, some commands as well as the data itself need to be sent. So the total time of communication should be longer. This results in further energy consumption and shortens the battery lifetime. And also, if there are many sensor nodes and the data is collected through multi-hop communication, a long period of time is needed for data collection. This leads to the limitation of the number of measuring points. These two facts motivate us to develop a new data compression method.

In the data compression method presented in this paper, the essential data, which is truly needed for estimating quasi-static displacement without accuracy deterioration, is extracted from the raw data. The amount of data of one epoch is compressed from 266 bytes to 28 bytes.

Fig.4 shows the format of the compressed data. The packet consists of the start flag (2bytes), acquisition time (4bytes), satellite ID (1byte), carrier phase (1byte) and check sum (2 bytes). The number of the satellite ID and the corresponding carrier phase is 


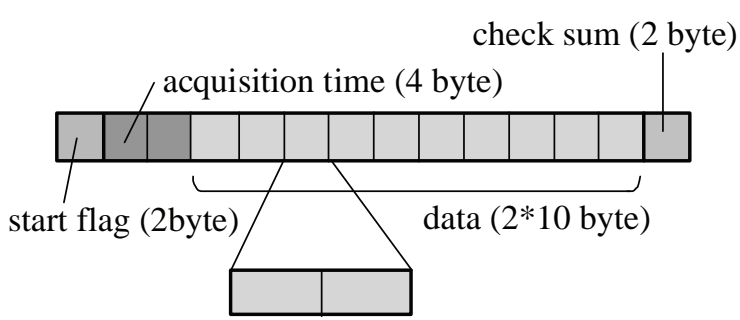

carrier phase (1byte) + satellite ID (1byte) $=2$ byte

Fig.4 Data format of the presented compression method ${ }^{11)}$.

fixed at 10 because a GPS receiver rarely observes more than 10 satellites at once. The CMR format contains other data than that shown in Fig.4, such as code pseudo-range and approximation position.

The most characteristic part of this data compression method is compression of the carrier phase to only 1 byte. The carrier phase is generally treated as double-precision data. The GPS receiver used in the prototype outputs the carrier phase as 4-byte data. The three most significant bytes represent the integer part of the carrier phase and the remaining 1 byte the fraction part. We extract only 1 byte corresponding to the decimal fraction. As mentioned in section 5, our target is quasi-static displacement. In this case, we can easily resolve the DD integer ambiguity. This means that the integer part of the carrier phase is not necessarily needed. Then, we can successfully compress the amount of data without diminishing the accuracy of displacement estimation ${ }^{11)}$.

\section{PERFORMANCE ASSESSMENT}

Experiments were conducted both in an open quarry site and in an ideal condition to investigate the performance of the prototype of the GWSN. The objectives of the experiment in the open quarry site were mainly focused on confirming that the system worked without serious system trouble and finding bugs hidden in the system. The experiment in the ideal condition was carried out to assess the accuracy of displacement monitoring.

\section{(1) Demonstration in an open quarry site}

The experiment was conducted in the open quarry site (Ohshu-shi, Iwate-prefecture) over 4 months from July to November 2009. The objectives were

1. to confirm that the system worked without any serious system trouble;

2. to check the performance of the wireless communication (distance and transmission rate);

3. to compare the resultant displacements between the existing system and the presented one;

4. to find out problems inherent in the system.

Photos $\mathbf{2}$ and $\mathbf{3}$ show an aerial view of sensor de-

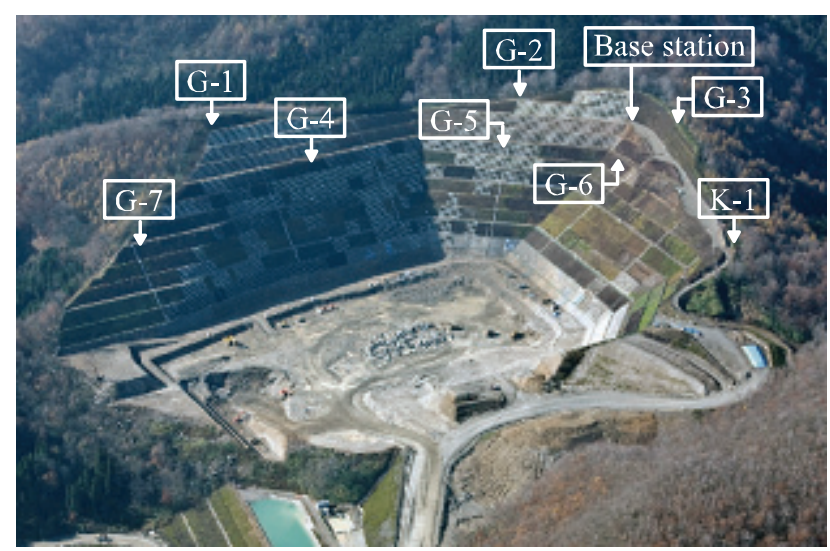

Photo 2 Aerial view of open quarry site.

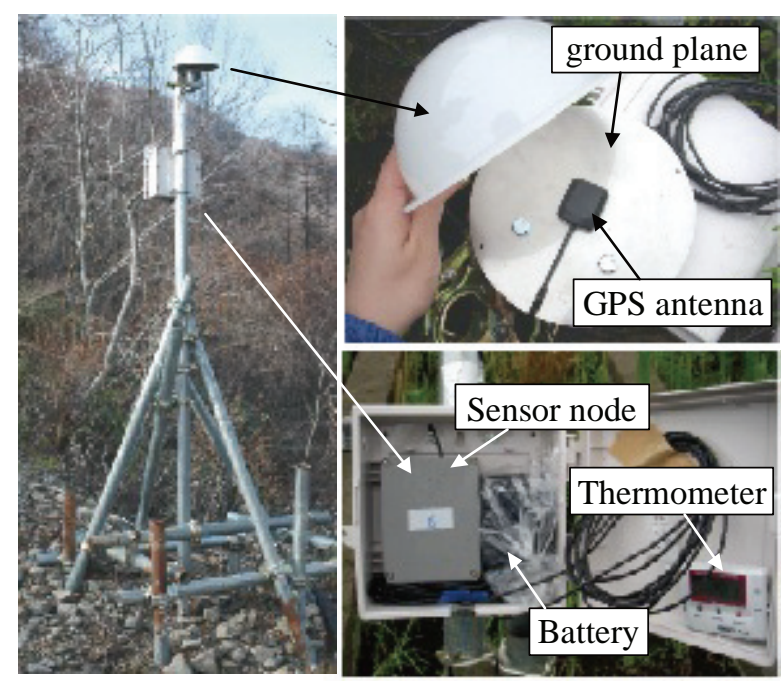

Photo 3 Sensor node installed at the site.

ployment in the open quarry site and the installed sensor node, respectively.

The existing GPS displacement monitoring system ${ }^{18)}$ had already been installed at the site. The existing system was supplied with electric power using cables. The wireless sensor nodes, developed by the authors, were fixed next to the existing sensors to compare the results. The symbol "K-1" in Photo 2 represents the reference point, and "G-1" to "G-7" the measuring points, whose relative positions from K-1 were monitored. In the data collection, raw GPS data was logged for 5 minutes at a $1 \mathrm{~Hz}$ sampling rate 4 times a day. Every sensor node had a nickel-hydride battery (22.8Wh). Considering this measurement schedule and the energy consumption of the sensor node, it was predicted that the sensor node could run for about 96 days without battery change.

The control PC was set at the base station in which the commercial AC power supply was available. The base station was visible from every measuring point. The distance in a straight line between the base sta- 


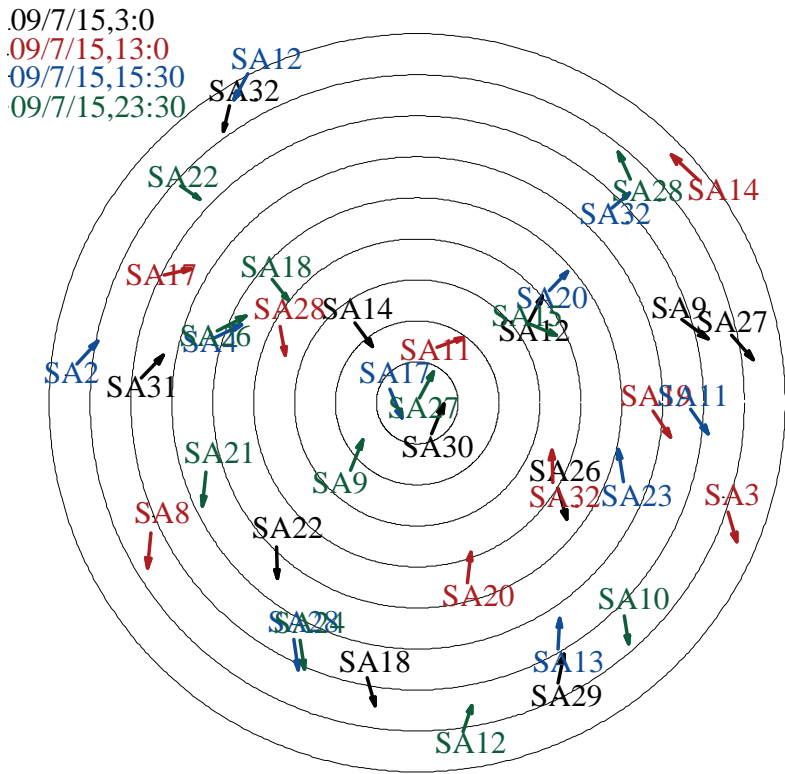

Fig.5 Satellite constellation in the observation period.

tion and the sensor node G-7, which corresponded to the longest base line, was about 504 meters. On the other hand, the shortest base line was 45 meters, between the base station and G-6.

The observation schedule was determined so that the satellite constellation uniformly covered the site. The satellite constellation of this experiment is shown in Fig.5. The circles represent the elevation angles. The center of the circle corresponds to a 90-degree elevation angle (the right overhead direction) and the largest circle represents 0 degrees (the horizon). The upward direction in Fig.5 corresponds to the northern direction. In this figure, there are no GPS satellites in the northern direction. This is because the orbit of the GPS satellite is designed to cover low latitudes. The arrows represent the orbits of the satellites in the duration of observation. The arrows are colored according to the segments. In this experiment, the satellites flying at the low elevation angle in the northern and western directions were unavailable due to the slope of the quarry.

\section{a) Running state and battery lifetime}

The lifetimes of the sensor nodes are listed in Table 1. The observations were carried out two times using fully charged batteries. The first experiment was conducted from 23 July to 13 October 2009 and the second from 13 October to 26 November 2009. The "end date" in Table 1 represents the final date of the data file saved on the central server. As shown in Table 1, most of the sensor nodes ran for more than 1 month, but the lifetimes were very different from each other. The average was about 50 days, which is almost half of the prediction (96 days). And also, the lifetime in the second experiment became shorter than that in the first one.
Table 1 Working days of sensor nodes in the quarry site.

\begin{tabular}{|c|c|c|c|c|}
\hline & \multicolumn{2}{|c|}{$\begin{array}{r}\text { The first trial } \\
(7 / 23-)\end{array}$} & \multicolumn{2}{|c|}{$\begin{array}{r}\text { The second trial } \\
(10 / 13-)\end{array}$} \\
\hline ID & End date & Days & End date & Days \\
\hline K-1 & $9 / 4$ & 44 & $11 / 21$ & 40 \\
\hline G-1 & $10 / 13$ & 83 & $11 / 26$ & 45 \\
\hline G-2 & $9 / 6$ & 46 & $11 / 21$ & 40 \\
\hline G-3 & $9 / 1$ & 41 & $10 / 31$ & 19 \\
\hline G-4 & $9 / 14$ & 54 & $11 / 21$ & 40 \\
\hline G-5 & $9 / 30$ & 70 & $11 / 26$ & 45 \\
\hline G-6 & $8 / 14$ & 23 & $11 / 17$ & 36 \\
\hline G-7 & $8 / 24$ & 33 & $11 / 23$ & 42 \\
\hline
\end{tabular}

Table 2 Data transmission rates in the first experiment.

\begin{tabular}{|c|c|c|c|c|}
\hline $\begin{array}{c}\text { Sensor } \\
\text { ID }\end{array}$ & $\begin{array}{c}\text { Distance } \\
{[\mathrm{m}]}\end{array}$ & $\begin{array}{c}\text { Number } \\
\text { of trials }\end{array}$ & $\begin{array}{c}\text { Number } \\
\text { of data }\end{array}$ & $\begin{array}{c}\text { Rates } \\
{[\%]}\end{array}$ \\
\hline K-1 & 211 & 175 & 175 & 100.0 \\
\hline G-1 & 411 & 329 & 329 & 100.0 \\
\hline G-2 & 117 & 137 & 137 & 100.0 \\
\hline G-3 & 60 & 161 & 161 & 100.0 \\
\hline G-4 & 324 & 214 & 213 & 99.5 \\
\hline G-5 & 122 & 278 & 274 & 98.6 \\
\hline G-6 & 45 & 88 & 88 & 100.0 \\
\hline G-7 & 504 & 130 & 126 & 96.9 \\
\hline
\end{tabular}

The main cause of the lifetime shortage is considered to be the high humidity condition. In this experiment, a thermohygrometer was placed in the box of the sensor node, and the temperature and humidity were sampled every 10 minutes. According to the measurement data, the humidity was over $90 \%$ most of the time. So the high humidity condition may have moistened the circuit board of the sensor node and decreased the electric resistance. This may have caused the shortage of the battery lifetime.

In order to examine the above hypothesis, we conducted a simple experiment in our laboratory using the same sensor nodes, which had been sufficiently dried after the experiment at the open quarry site. In this experiment, two sensor nodes were placed in boxes in dry condition and the other two into other boxes in wet condition. The control PC collected GPS data from the sensor nodes according to the same schedule as in the quarry site. Results show that the two sensor nodes in dry condition of less than $10 \%$ humidity worked for 106 and 110 days, respectively. On the other hand, the two sensor nodes in wet condition of $100 \%$ humidity worked 
only for 46 and 59 days. This suggests that a high humidity condition shortens the battery lifetime.

\section{b) Wireless communication distance and data transmission rate}

Table 2 presents the data transmission rates of each sensor node in the first experiment. The number of trials can be approximately calculated by multiplying the working days by four, since raw GPS data was collected 4 times a day. The number of data in Table 2 is the number of data files saved in the control PC. The rates represent the ratio of the number of saved data files to trials.

In this experiment, the data sets were almost $100 \%$ transmitted to the control PC. In the prototype system, the control PC is designed to keep sending "data transmission command" to the sensor nodes up to time out. Therefore, once the sensor node receives a packet from the control PC, it can transmit the whole data set of a segment to the control PC. On the other hand, if the sensor node does not receive a packet until time out, the control PC does not collect the corresponding data set. This is one of the issues to be improved in future works.

\section{c) Comparison with the existing displacement monitoring system}

In this experiment, we fixed our sensor nodes next to the existing GPS sensors to compare the results of displacement monitoring.

Fig 6. presents the time series of displacements measured at the sensor node G-1. The figures correspond to the NS (North-South), EW (East-West) and UD (Up-Down) components from top to bottom, respectively. The horizontal and vertical axes represent the accumulation time [days] and displacement $[\mathrm{cm}]$. The dots and broken lines represent the results of the existing system. The existing system outputs displacement every hour (dot) and estimates the smoothed curve by applying trend analysis (broken line). The triangles and solid lines are the results obtained from the presented system. The triangles are plotted with a 1-day interval since the displacement of the presented system is estimated using 4 segments together, while the solid line represents their moving average over 5 days. In the graphs, there are missing data for the existing system from 6 to 16 days. In this period, the displacements of all measuring points were not monitored due to a power supply fault.

Comparison of the results of the existing and the presented systems indicates that the trends of estimated displacements are similar to each other. So the presented system seems to work well in this experiment. However, some small discrepancies appear in the NS and UD direction. For example, the data for the 11th day in the UD direction includes a relatively large estimation error of about $1.5 \mathrm{~cm}$. The main
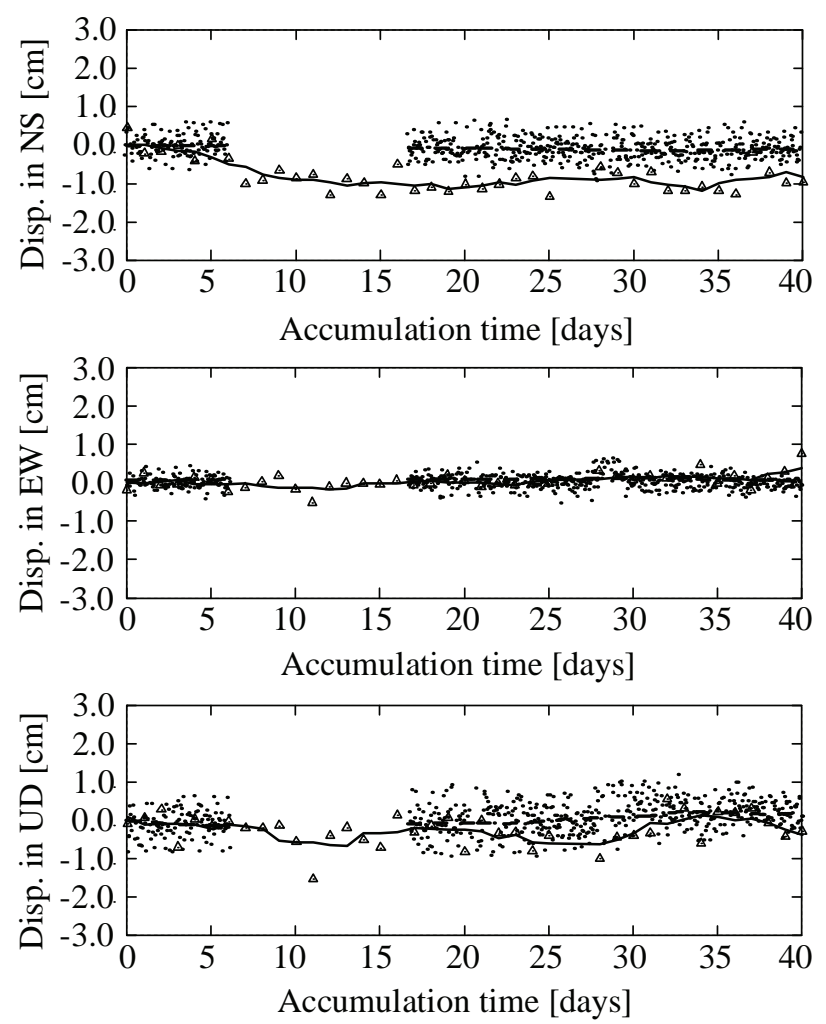

Fig. 6 Comparison between the displacements monitored by the existing and the presented system at the sensor G-1.

cause of this error is the antenna noise. In the present system, a small patch antenna is used to minimize the cost of the sensor node. However, such a small patch antenna is generally never used for displacement monitoring because it is very weak against the multi-path noise. It is known that the multi-path noise behaves almost the same way every day because the geometrical condition between GPS satellites and a sensor is almost the same every day too. Therefore, in the analysis, the multi-path noise was first estimated using the data collected on the first day and the multi-path noise included in the following measurements were subtracted using the estimated noise. However, the multi-path noises included in the raw data sometimes behaved in a very different manner. That is why the multi-path noise was unsuccessfully canceled and the estimated displacement includes a relatively large error.

\section{d) Summary of demonstration in the quarry site}

We conducted an experiment in the open quarry site and the results show that the presented system runs without serious system trouble and is able to monitor displacement. On the other hand, this experiment clarifies some problems to be solved:

1) The need to develop a box for protecting the sensor node from a high humidity environment and to verify the battery lifetime.

2) The need to implement a more reliable data transmission method. 
3) The need to develop an estimation method of multi-path noise and investigate the term of validity of the estimated noise.

\section{(2) Demonstration in an ideal condition}

In the experiment conducted in the open quarry site, we could not assess the accuracy of displacement monitored by the presented system because nobody knew the actual displacement. Therefore, we carried out an experiment in the well-known condition, which means that we intentionally moved the GPS antenna using an accurate movable stage which is able to control 3D displacement with an accuracy of a few micrometers.

\section{a) Outline of experiment}

In this experiment, one of the GPS antennas was fixed on top of the movable stage, as shown in Photo 4. The other antenna was placed on a plastic box as a reference point. Both antennas were attached to the ground plane to suppress multi-path noise. These two antennas were fixed at different heights so that the data might include the different multi-path noises. The experiment was carried out in an ideal condition, where there were no obstacles over the site. The observation period was about 40 days from 26 December 2009 to 3 February 2010. In the experiment, the movable stage kept its position during the first 2 days. After that, it vertically went down by $1 \mathrm{~cm}$ in 10 days at the constant speed. In the next 10 days, it moved to the northern direction by $1 \mathrm{~cm}$ and after that to the western direction by $1 \mathrm{~cm}$ in the following 10 days. After the 32 days mentioned above, the movable stage stood still for 9 days. The raw GPS data (carrier phase) was observed for 5 minutes with a 1 $\mathrm{Hz}$ sampling rate every hour. However, the data acquisition started 4 minutes earlier every day to equalize the satellite constellation. In the experiment, 23 segments were collected every day.

\section{b) Analysis method}

The displacements were estimated by picking out some segments from the 23 and analyzing them together, as mentioned in section 3.(3). Fig.7 shows the displacement time series estimated using all segments. The graphs represent the displacement in the NS, EW and UD directions and the total number of satellites used in the analysis from top to bottom, respectively. The solid and broken lines represent the analyzed results and the actual displacement controlled by the movable stage, respectively. The analyzed results are missing on the third day because the corresponding raw GPS data was not collected due to wireless communication trouble.

In the case of analyzing 23 segments together, the 3-directional displacement was estimated accurately and the doubled standard deviation from the actual

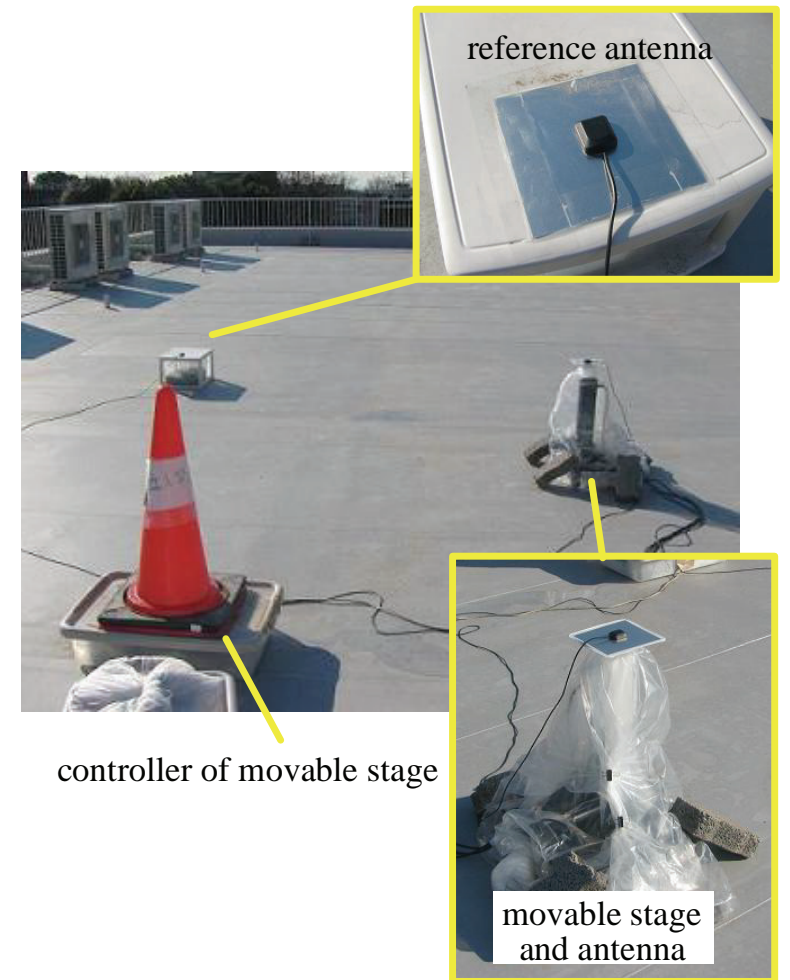

Photo 4 Experimental setup using the movable stage in an ideal condition.
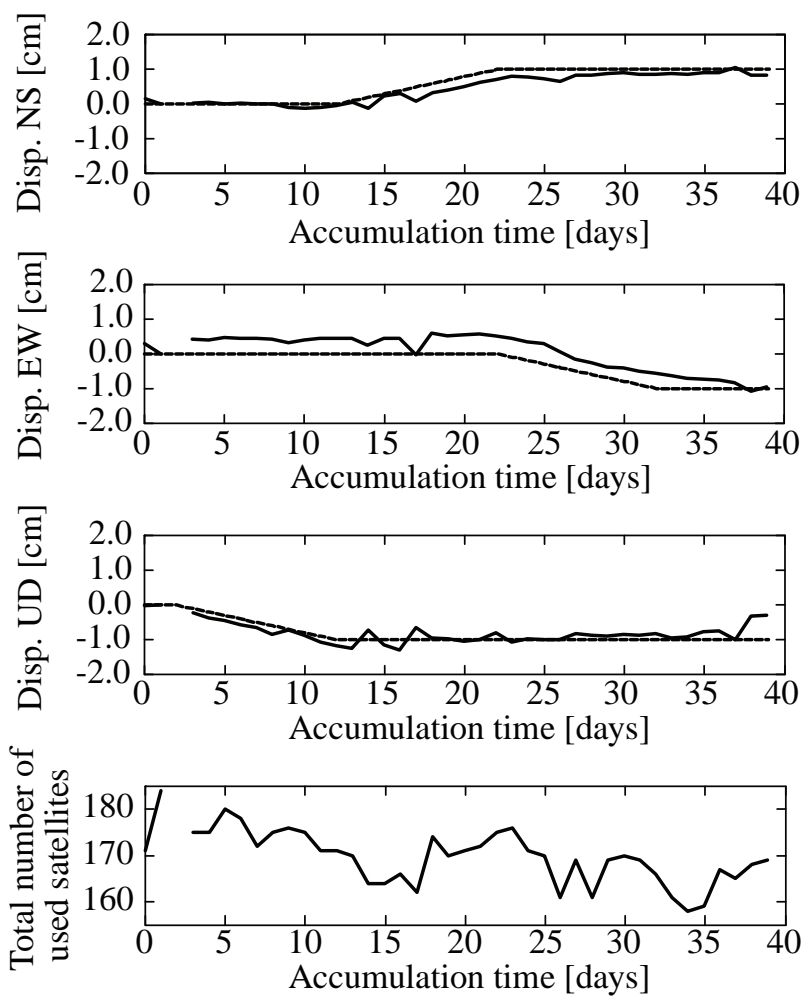

Fig.7 Time series of displacement estimated using 23 segments.

displacement was about 2.8 and 2.2 [mm] in horizontal and vertical directions, respectively. This result indicates that the presented system has a potential ability of monitoring ground displacement with sub-centimeter accuracy. However, in order to decrease the energy consumption and make the battery 
lifetime longer, the observation period in a day needed to be decreased. So, in the following subsections, how to decrease the use of segments without diminishing the accuracy is discussed.

\section{c) Effect of segment selection on accuracy}

We investigated the relationship between the choice of segments and the accuracy of displacement by selecting 1 to 3 segments from the 23. Fig.8 shows the results for the case of analyzing only 1 segment. The vertical axes of the graphs represent the accuracy (doubled standard deviation), number of used satellites, PDOP (Position Dilution of Precision) and condition number of the coefficient matrix of the normal equation, respectively. The horizontal axis represents the segment ID number. In the analysis, a time series of 3-directional displacement was calculated for each single segment. The accuracy of each segment was evaluated by calculating the doubled standard deviation from the actual displacement. The number of satellites, PDOP and condition number were obtained by averaging their estimated values over 40 days.

As shown in Fig.8, if the PDOP or condition number is relatively small, the accuracy tends to be low. The accuracy in the case of using segments \#3, \#5, \#9 and \#12 is better than for the others. Since the value of PDOP can be predicted without collecting raw GPS data on-site, we can possibly make a reasonable observation schedule with the PDOP. However, the accuracy is only $2.0 \mathrm{~cm}$ even if the best segment is selected in the case of using a single segment. Therefore more segments must be analyzed together to improve the accuracy. The time series of displacement estimated using segment \#5 is described in Fig.9 for reference. The estimated displacement is relatively inaccurate compared with the result when using 23 segments, as shown in Fig.7.

At the next step, we considered the case of analyzing two segments together. In this analysis, segment \#5 was always used and the other segment was selected from the other 22 segments. The relationship between the choice of segments and the accuracy in the case of analyzing two segments is described in Fig.10. The accuracy tends to be good in the case of a small PDOP, but the correlation is not very clear. A smaller value of PDOP or condition number does not necessarily yield better accuracy. For example, the combination of segments \#5 and \#11 yields a relatively large PDOP and condition number but gives good accuracy. The main cause is considered to be the influence of observation noise. The accuracy depends on the observation noise as well as the quality of the coefficient matrix.

As shown in Fig.8 and Fig.10, the accuracies when using segments \#4, \#7, \#10, \#15 and following are
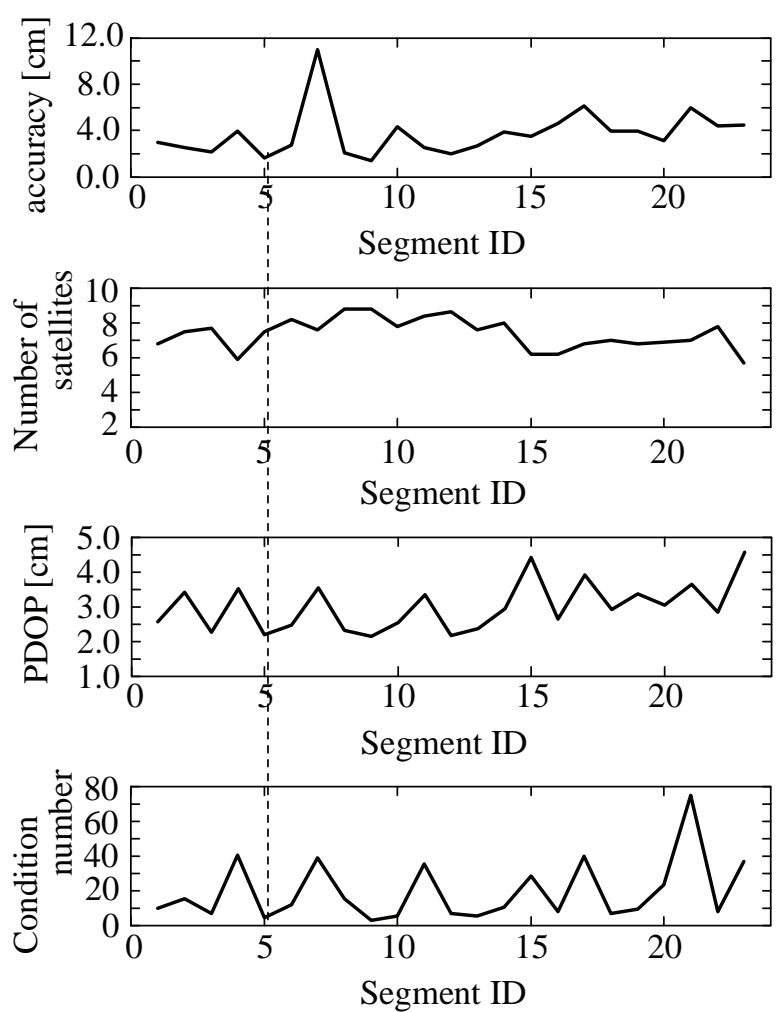

Fig.8 Relationship between the choice of segments and the accuracy in the case of analyzing a single segment.
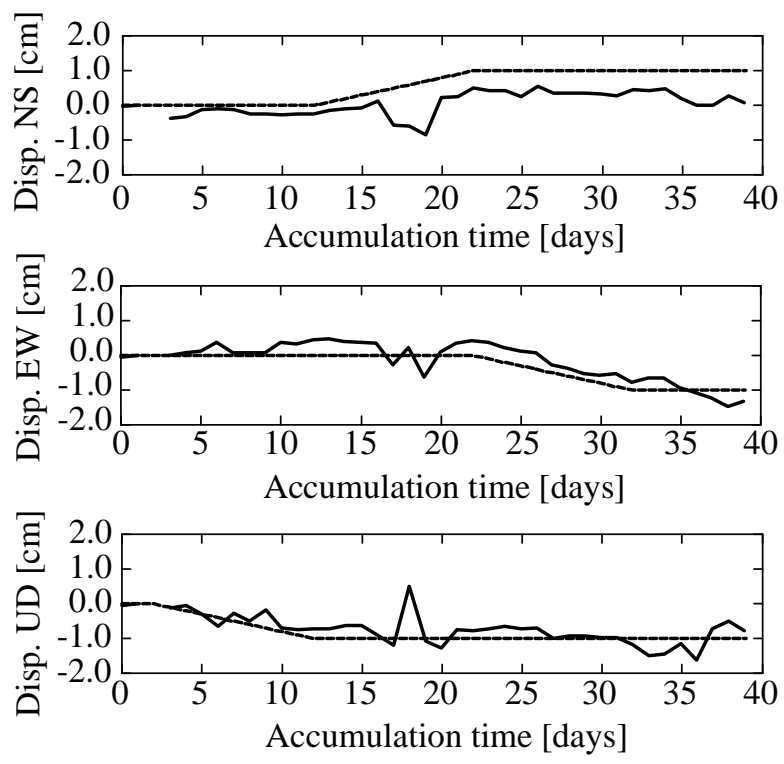

Fig.9 Time series of displacement estimated using segment \#5.

relatively worse than the others. This fact shows that these segments do not improve the accuracy even if more than two segments are used.

Fig.11 describes the time series of displacement estimated using segments \#5 and \#8. This combination gives the smallest PDOP value. In this case, the doubled standard deviation is about 1.0 [cm], which is an improvement from the result of a single segment.

In order to improve the accuracy further, three- 


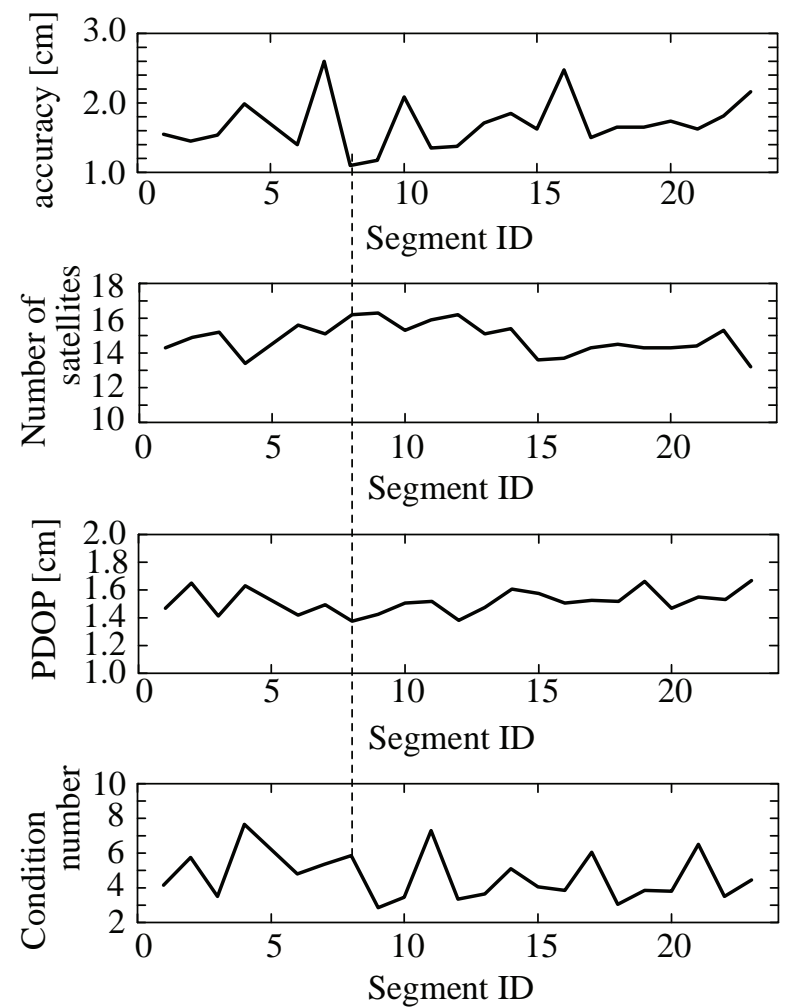

Fig.10 Relationship between the choice of segments and the accuracy in the case of analyzing two segments.
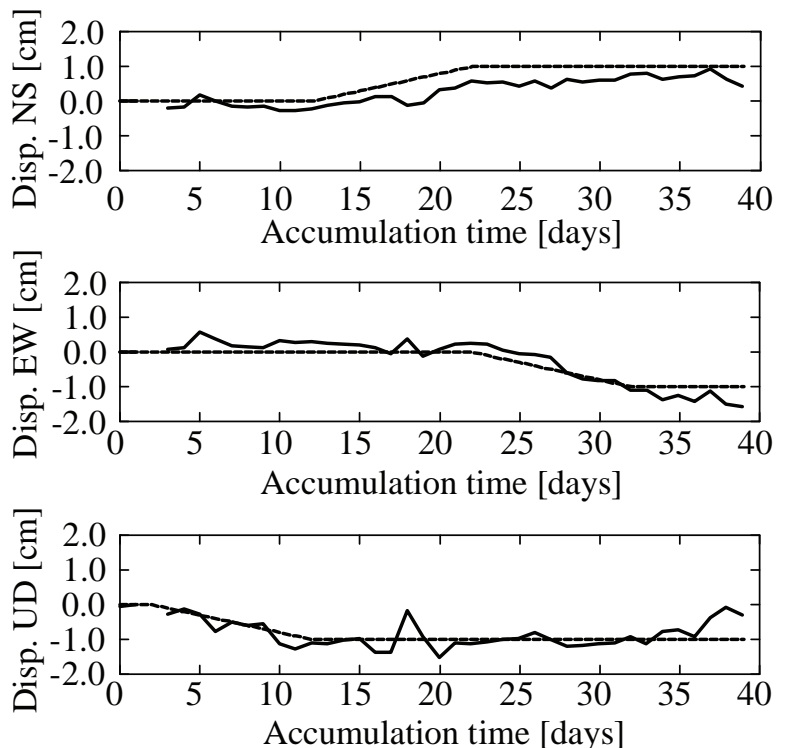

Fig.11 Time series of displacement estimated using segments \#5 and \#8.

segment combinations were tested. In this analysis, segments \#5 and \#8 were always used and the other segment was added from the other 21 . The results are shown in Fig.12. In this analysis, the accuracy of all combinations seems to be improved due to the additional segments. Therefore it can be said that the analysis with multiple segments is able to prevent serious accuracy deterioration. The addition of segments \#2, \#9, \#15 or \#17 especially improves the accuracy. However, the PDOP and condition number
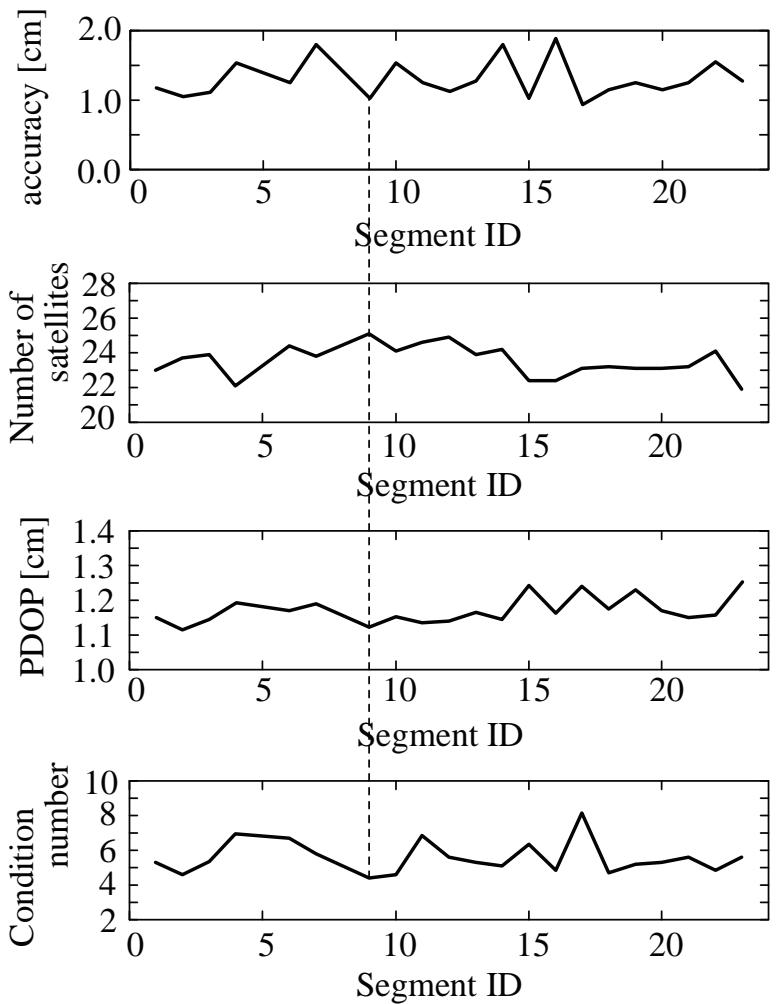

Fig.12 Relationship between the choice of segments and the accuracy in the case of analyzing three segments.
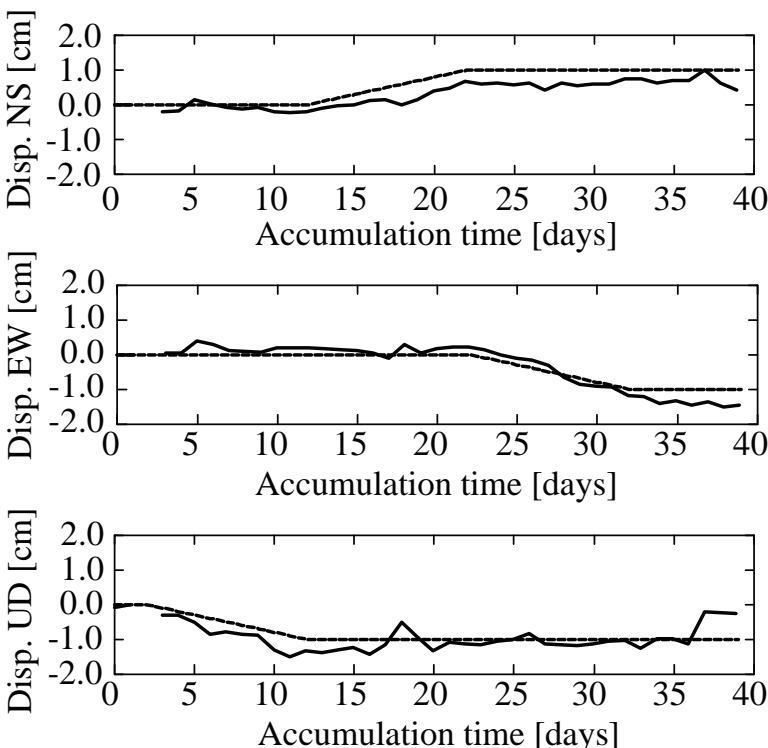

Fig.13 Time series of displacement estimated using segments \#5, \#8 and \#9.

of the results of \#15 and \#17 are not as small, so that it is difficult to predict the accuracy improvement from before the analysis.

Fig.13 describes the time series of displacement in the case of analyzing segments \#5, \#8 and \#9 together. Comparing the results shown in Fig.9, Fig.11 and Fig.13 shows that the behavior of the estimated displacement is gradually getting close to the actual displacement. 


\section{d) Summary of demonstration in an ideal condi- tion}

We conducted an experiment in an ideal condition to evaluate the accuracy of the system presented in this paper. In the experiment, one GPS antenna was fixed on top of a movable stage, which was able to control the displacement with an accuracy of a few micrometers. We also tested whether different segment combinations improved the accuracy. The results of the experiment indicate the following:

1) The analysis using 23 segments has the potential of monitoring displacements with sub-centimeter accuracy in an ideal condition.

2) The accuracy in the case of using three segments can be comparable to the case of using 23 segments if the best combination is selected.

3) The index of the PDOP or condition number was helpful in selecting the best segment when we analyzed displacement with a single segment. But it is difficult to predict the best segment combination using those indexes. This fact implies that the accuracy depends on the observation noise rather than the quality of the coefficient matrix in the case of multiple segment usage.

\section{SUMMARY}

The authors have developed a displacement monitoring system using the technologies of a low-cost L1 GPS and wireless sensor network. The use of these technologies enables reduction of the total cost of displacement monitoring because of the low-cost sensor node and the workability of the system.

In this research, we developed a prototype system and demonstrated it in an open quarry site. The results of this experiment indicate that the system runs without serious system trouble in an outdoor environment. Areas for future works were found. We also tested the accuracy of displacement by conducting an experiment in an ideal condition using a movable stage. The results show that the accuracy can be improved to the sub-centimeter level.

The future works are to implement protection against high humidity, to reconstruct the network protocol of collecting data and to improve the displacement analysis method, such as the noise estimation method and the choice of the best segment combination.

\section{REFERENCES}

1) Lynch, J. P.: Decentralization of wireless monitoring and control technologies for smart civil structures, Ph.D. thesis, Department of Civil and Environmental Engineering,
Stanford University, Stanford, CA., 2002.

2) Nagayama, T., Spencer, Jr. B. F. and Fujino, Y.: Smart sensor middleware development for dense structural vibration measurement, Journal of JSCE A, Vol. 65, No. 2, pp. 523-535, 2009. (in Japanese)

3) Sakata, S.: Ubiquitous Technology, Sensor Network, Ohmsha, 2006. (in Japanese)

4) Kondo, H., Cannon, E., Shimizu, N. and Nakagawa, K.: Developments of a ground displacement monitoring system by using the global positioning system, Journal of JSCE, No.546/VI-32, pp.157-168, 1996. (in Japanese)

5) Matsuda, H., Adachi, H., Nishimura, Y. and Shimizu, N.: Applicability of the trend model for smoothing measured displacements by using global positioning system and methods for predicting displacement behavior, Journal of JSCE, No.715/III-60, pp. 333-343, 2002. (in Japanese)

6) Shimizu, N.: Continuous Displacement Monitoring using Global Positioning System for Assessment of Slope Stability, the 2nd Southeast Asian Workshop on Rock Engineering, Hanoi, pp.136-145, 2003.

7) Seynat, C., Hooper, G., Roberts, C. and Rizos, C.: Low-cost deformation measurement system for volcano monitoring, Proceedings of the 2004 International Symposium on GNSS/GPS, 2004.

8) Sadler, C. M. and Martonosi, M.: Data Compression Algorithms for Energy-Constrained Devices in Delay Tolerant Networks, Proc., the ACM Conference on Embedded Networked Sensor Systems (SenSys'06), November 1-3, 2006.

9) Talbot, N. C.: Compact data transmission standard for high-precision GPS, 9th Int. Tech. Meeting of the Sat. Div. of the U.S. Inst. of Navigation, Kansas City, Missouri, 17-20, Sept., pp.861-871, 1996.

10) Heo, Y., Yan, T., Lim, S. and Rizos, C.: International Standard GNSS Real-Time Data Formats and Protocols, Proceedings of IGNSS Symposium, Holiday Inn Surfers Paradise, Australia, Dec., 2009.

11) Kosaka, T. and Saeki, M.: Data reduction method for L1 GPS receivers of wireless sensor network system, Journal of Applied Mechanics, Vol.9, JSCE, pp.709-716, 2006. (in Japanese)

12) Sawada, M., Shiba, Y. and Saeki, M.: Development of displacement monitoring system and GPS wireless sensors, Report of Taisei Technology Center, Vol.43, 2010. (in Japanese)

13) Hofmann-Wellenhof, B., Lichtenegger, H. and Collins, J.: GPS, Theory and Practice, SpringerWienNewYork, 2001.

14) Gassner, G., Wieser, A. and Bruvver, F.: GPS Software development for monitoring of landslides, Proc., FIG XXII Congress (CD-ROM), 2002.

15) Saeki, M., Kaneko, S. and Inoue, T.: Development of GPS positioning algorithm based on static and dense deployment conditions, Journal of Applied Mechanics, Vol.10, JSCE, pp.639-648, 2007. (in Japanese)

16) Saeki, M., Inoue, T., Sawada, M. and Shiba, Y.: Fundamental study on the accuracy of displacement monitoring of GPS wireless sensor network, Journal of Applied Mechanics, Vol.12, JSCE, pp.975-982, 2009. (in Japanese)

17) MU-1 manual (Website of circuit design INC.), http://www.circuitdesign.jp/jp/products/manual/MU-1_120 0.pdf (in Japanese)

18) Website of shamen-net.com, http://www.shamen-net.com/ shamen01.html

(Received September 18, 2012) 